\title{
Phenotypic Evaluation of Prevalence of Metallo-Beta-Lactamase (MBL) Production among Clinical Isolates of Pseudomonas aeruginosa and Acinetobacter Species in a Tertiary Care Hospital of North India
}

\author{
Noor Jahan ${ }^{1}$, Razia Khatoon ${ }^{2} *$ and Mohd Rashid ${ }^{3}$ \\ ${ }^{1}$ Department of Microbiology, Integral Institute of Medical Sciences and Research, Integral \\ University, Lucknow-226026, India \\ ${ }^{2}$ Department of Microbiology, Hind Institute of Medical Sciences, Mau, Ataria, Sitapur- \\ 261303, India \\ ${ }^{3}$ Department of Microbiology, F.H. Medical College, Tundla, Agra-283204, India \\ *Corresponding author
}

\begin{abstract}
A B S T R A C T
Pseudomonas aeruginosa and Acinetobacter species have emerged as important Keywords nosocomial pathogens. Carbapenems had been the drugs of choice for penicillin \& cephalosporin resistant Pseudomonas and Acinetobacter species infections. However, this scenario has changed with the emergence of Metallo-beta-lactamase (MBL) producing strains as these enzymes hydrolyze all beta-lactams, thereby, increasing patient morbidity Pseudomonas aeruginosa, Acinetobacter species, Metallobeta-lactamase (MBL), ImipenemEDTA combined disk test (CDT), Modified Hodge test (MHT).

Article Info

Accepted:

15 June 2018 Available Online: 10 July 2018 and mortality. This situation prompts early and accurate detection of MBL producers. Hence the present study was done to phenotypically evaluate the prevalence of MBL production among 235 clinical isolates of Pseudomonas aeruginosa (183) and Acinetobacter species (52). The antimicrobial susceptibility testing was done by Kirby Bauer disk diffusion method. About $26.4 \%$ were found to be resistant to carbapenems tested. These screen positive isolates gave results of positive MBL production among $79.0 \%$ and $59.7 \%$ isolates by imipenem-EDTA combined disk test and modified Hodge test respectively. The prevalence of MBL production was found to be $21.9 \%$ and $17.3 \%$ among isolates of Pseudomonas aeruginosa and Acinetobacter species respectively. The prevalence was found to be higher among isolates from inpatients (26.1\%) in comparison to those from outpatients $(9.5 \%)$. Most of the MBL producers were isolated from pus samples $(33.7 \%)$, followed by sputum (18.6\%). The in vitro antimicrobial susceptibility profile of MBL producers showed that they were multidrug resistant, being $100 \%$ sensitive only to colistin and polymyxin B. To conclude, detection of MBL producers should be routinely done in all microbiological laboratories along with implementation of strict infection control policies and antibiotic stewardship for better patient management.
\end{abstract}

\section{Introduction}

Pseudomonas and Acinetobacter species have emerged as important nosocomial pathogens.
They are widely distributed in nature and their presence in the hospital environment puts debilitated patients, especially those in intensive care units (ICUs) at risk of 
opportunistic infections by these multidrug resistant pathogens (Sarkar et al., 2006). Carbapenems had been the drugs of choice for penicillin and cephalosporin resistant Pseudomonas and Acinetobacter species infections, because of their broad spectrum activity and stability to hydrolysis by most of the beta-lactamases including extended spectrum beta lactamases (ESBLs). However, this scenario has changed with the emergence of Metallo-beta-lactamase (MBL) producing strains (Varaiya et al., 2008; Ahir et al., 2012; Shivappa et al., 2015).

Metallo-beta-lactamases (MBL) are metalloenzyme of Ambler class B which require divalent cations of zinc as cofactors for enzyme activity and are inhibited by metal chelators like ethylene diamine tetra acetic acid (EDTA) and thiol-based compound but not by sulbactam, tazobactam and clavulanic acid (Purohit et al., 2012). The MBLs efficiently hydrolyze all beta-lactams, except monobactam i.e. aztreonam (Galani et al., 2008). The genes for MBL production (IMP and VIM) are horizontally transferable via plasmids and can rapidly spread to other bacteria (Senda K et al., 1996; Bennett, 1999). Several studies have reported global increase in the prevalence of MBL producing nonfermenting bacilli (Varaiya et al., 2008; Saha $\mathrm{R}$ et al., 2010; Deshmukh et al., 2011). MBL production is typically associated with resistance to aminoglycosides and fluoroquinolones, further compromising the therapeutic options (Purohit et al., 2012). Thereby making it a matter of concern with regard to the future of antimicrobial chemotherapy (Bush et al., 1995).

This situation prompts an early and accurate detection of MBL producing organisms of crucial importance. Carbapenemase gene detection by molecular methods is the gold standard, but is available in only few reference laboratories, therefore, phenotypic tests have been developed for detection of MBL producers in clinical laboratories (Andre et al., 2012). Therefore, we did this study to evaluate the prevalence of metallo-beta-lactamase (MBL) producing Pseudomonas aeruginosa and Acinetobacter species derived from clinical samples at our Tertiary care hospital by using phenotypic methods.

\section{Materials and Methods}

A hospital based prospective study was done over a period of 1 year from January to December 2017. The study was approved by Institutional Ethics Committee. Various clinical samples such as pus, urine, blood, sputum and catheter tip, received in bacteriology laboratory of department of Microbiology, from both outpatient departments (OPD) and inpatient departments (IPD including patients admitted in various wards and intensive care units) were cultured on Blood agar and MacConkey agar and incubated aerobically at $37^{\circ} \mathrm{C}$ for 24 hours and the growth was identified as per the standard microbiological protocols and procedures (Crichton, 2006). A total of 235 consecutive, non-duplicate isolates of Pseudomonas aeruginosa $(\mathrm{N}=183)$ and Acinetobacter species $(\mathrm{N}=52)$ were included in the study. All gram positive and other Gram negative bacterial isolates were excluded. Antimicrobial susceptibility testing was performed on Mueller-Hinton agar (HiMedia Laboratories, Mumbai, India) by Kirby-Bauer disk diffusion method according to Clinical and Laboratory Standards Institute (CLSI) guidelines using antibiotics (HiMedia Laboratories, India) such as, amikacin $(30 \mu \mathrm{g})$, gentamicin $(10 \mu \mathrm{g})$, piperacillin $(100 \mu \mathrm{g})$, piperacillin/tazobactam $\quad(100 / 10 \mu \mathrm{g})$,

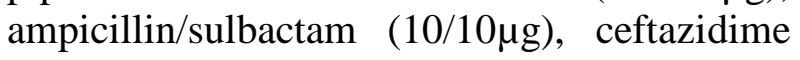
$(30 \mu \mathrm{g}), \quad$ cefotaxime $(30 \mu \mathrm{g})$, ceftriaxone $(30 \mu \mathrm{g})$, cefepime $(30 \mu \mathrm{g})$, imipenem $(10 \mu \mathrm{g})$, meropenem $(10 \mu \mathrm{g}), \quad$ colistin $(10 \mu \mathrm{g})$, polymyxin B (300 units), ciprofloxacin $(5 \mu \mathrm{g})$ 
and aztreonam (30 $\mu \mathrm{g})$. Pseudomonas aeruginosa ATCC 27853, Klebsiella pneumoniae ATCC BAA-1705 (Modified Hodge Test positive) and Klebsiella pneumoniae ATCC BAA-1706 (Modified Hodge Test negative) were used as quality control strains (CLSI, 2016).

\section{Screening method for MBL production}

As mechanisms of resistance can be different for imipenem and meropenem, therefore, resistance to imipenem is not always predictive of resistance to meropenem, and vice versa (Jones et al., 2006). Hence, isolates resistant to imipenem (zone size $\leq 15 \mathrm{~mm}$ for Pseudomonas aeruginosa and $\leq 18 \mathrm{~mm}$ for Acinetobacter species) and / or meropenem (zone size $\leq 15 \mathrm{~mm}$ for Pseudomonas aeruginosa and $\leq 14 \mathrm{~mm}$ for Acinetobacter species) were considered as screening positive (CLSI, 2016). These isolates were then subjected to two different phenotypic tests for confirmation i.e. Imipenem-EDTA combined disk test and modified Hodge test.

\section{Imipenem - EDTA Combined disk test (CDT)}

One $10 \mu \mathrm{g}$ imipenem disk alone along with another $10 \mu \mathrm{g}$ imipenem disk supplemented with $750 \mathrm{mg}$ EDTA (Hi-Media Lab, India) were placed at a distance of $20 \mathrm{~mm}$ from center to center on lawn culture of the test organism on Mueller Hinton agar and incubated at $35^{\circ} \mathrm{C}$ for $16-18 \mathrm{hrs}$ (Altun et al., 2013; El-Din et al., 2014). The inhibition zones of imipenem and imipenem EDTA was compared and if zone of inhibition of imipenem-EDTA disk was $\geq 7 \mathrm{~mm}$ more than that of imipenem disk alone, it was considered as MBL producer (Figure 1).

\section{Modified Hodge Test (MHT)}

A 0.5 McFarland standard suspension of Escherichia coli ATCC 25922 was prepared in broth. The surface of a Mueller Hinton agar plate was inoculated evenly with 1:10 dilution of the suspension in broth using a sterile swab as for the routine disk diffusion procedure. After drying the plate for $15 \mathrm{~min}$ imipenem disk $(10 \mu \mathrm{g})$ was placed at the center of the plate and the imipenem resistant test strains from the overnight culture plates along with the positive (Klebsiella pneumoniae ATCC BAA-1705) and negative (Klebsiella pneumoniae ATCC BAA-1706) control for MHT were streaked heavily from the edge of the disk to the periphery of the plate (Lee $e t$ al., 2001). The presence of a distorted inhibition zone of imipenem after overnight incubation was interpreted as modified Hodge test positive (Figure 2).

\section{Statistical analysis}

The collected data were statistically analyzed using SPSS software, Chicago, version 16. The association between MBL production and resistance to antibiotics was analyzed using Chi-square test and $\mathrm{p}$ value $<0.05$ was considered as statistically significant.

\section{Results and Discussion}

A total of 235 isolates of Pseudomonas aeruginosa $(\mathrm{N}=183)$ and Acinetobacter species $(\mathrm{N}=52)$ isolated from various clinical samples were screened for MBL production as shown in Table 1. This finding was found to be statistically significant $(\mathrm{p}<0.001)$. Out of these 235 clinical isolates, 26.4\% (62/235) were found to be resistant to carbapenems tested (imipenem and / or meropenem) and hence were screening test positive for MBL production, with $67.7 \%$ Pseudomonas aeruginosa and $32.3 \%$ Acinetobacter species as depicted in Figure 3. These screen positive isolates on being subjected to phenotypic confirmatory tests yielded positive MBL production among $79.0 \%(49 / 62)$ and $59.7 \%$ (37/62) isolates by CDT and MHT respectively, this difference was found to be 
statistically significant $(\mathrm{p}<0.001)$ as depicted in Table 2. It was found that the prevalence of MBL production was higher among isolates of Pseudomonas aeruginosa $(21.9 \%, 40 / 183)$ as compared to those among Acinetobacter species $(17.3 \%, 9 / 52)$ as shown in Table 3. However, this difference was not found to be statistically significant $(\mathrm{p}=0.476)$. Also, the prevalence of MBL producing organisms was found to be higher among isolates from inpatients $(26.1 \%, 42 / 161)$ in comparison to those from outpatients $(9.5 \%, 07 / 74)$ as depicted in Table 4, this difference was also found to be statistically significant $(\mathrm{p}=$ 0.004). Table 5 shows that most of the MBL producers were isolated from pus samples (33.7\%), followed by sputum (18.6\%), and least from blood $(4.8 \%)$. This finding was found to be statistically significant $(\mathrm{p}=$ 0.002).

The in vitro antimicrobial susceptibility profile of 183 isolates of Pseudomonas aeruginosa and 52 isolates of Acinetobacter species showed that MBL producers possessed multidrug resistance with highly decreased susceptibility to piperacillin, piperacillin/ tazobactam, ceftazidime, cefepime, amikacin, gentamicin as well as to ciprofloxacin. All the
MBL producers were found to be highly resistant to imipenem (100\%) and meropenem $(100 \%)$, and highly sensitive to colistin $(100 \%)$ and polymyxin B (100\%). The susceptibility pattern of MBL producers for most of the tested drugs was found to be statistically significantly different $(\mathrm{p}<0.05)$ as compared to non-MBL producers as depicted in Table 6 and 7.

In the present study majority of the isolates were of Pseudomonas aeruginosa (77.9\%) as compared to Acinetobacter species (22.1\%). A very high percentage of Pseudomonas aeruginosa were isolated from pus samples (91.3\%), followed by urine $(87.2 \%)$ and least from sputum (55.8\%), whereas, majority of Acinetobacter species were isolated from sputum $(44.2 \%)$ followed by catheter tip (37.5\%), and least from pus (8.7\%). However, in contrast to our finding a study from Mysore detected very high percentage of Pseudomonas (36\%) and Acinetobacter (32\%) isolated from endotracheal tube suction samples followed by pus samples $(11.7 \%$ and $4.94 \%$ respectively) and very low percentage isolated from urine samples $(0.24 \%$ and $0.35 \%$ respectively) (Shivappa et al., 2015).

\begin{tabular}{|c|c|c|c|}
\hline \multicolumn{4}{|c|}{ Table.1 Distribution of organisms isolated from various clinical samples $(\mathbf{N}=\mathbf{2 3 5})$} \\
\hline Samples tested & $\begin{array}{l}\text { Pseudomonas } \\
\text { aeruginosa, N (\%) }\end{array}$ & $\begin{array}{l}\text { Acinetobacter } \\
\text { species, N }(\%)\end{array}$ & $\begin{array}{l}\text { Chi-Square }\left(\chi^{2}\right) \\
\text { and } * \text { p value }\end{array}$ \\
\hline Pus $(\mathbf{N}=92)$ & $84(91.3 \%)$ & $08(8.7 \%)$ & \multirow{6}{*}{$\begin{array}{l}\chi^{2}=30.084, \\
p<0.001\end{array}$} \\
\hline Urine $(\mathrm{N}=47)$ & $41(87.2 \%)$ & $06(12.8 \%)$ & \\
\hline $\operatorname{Blood}(\mathbf{N}=21)$ & $14(66.7 \%)$ & $07(33.3 \%)$ & \\
\hline Sputum $(\mathbf{N}=43)$ & $24(55.8 \%)$ & $19(44.2 \%)$ & \\
\hline Catheter Tip $(\mathrm{N}=32)$ & $20(62.5 \%)$ & $12(37.5 \%)$ & \\
\hline Total $(\mathbf{N}=\mathbf{2 3 5})$ & $183(77.9 \%)$ & $52(22.1 \%)$ & \\
\hline
\end{tabular}


Table. 2 Comparative evaluation of MBL production among screen positive isolates $(\mathrm{N}=62)$ by using Imipenem-EDTA combined disk test (CDT) and Modified Hodge test (MHT)

\begin{tabular}{|c|c|c|c|c|}
\hline \multirow[t]{2}{*}{ CDT } & \multicolumn{3}{|l|}{ MHT } & \multirow{2}{*}{$\begin{array}{l}\text { Chi-Square }\left(\chi^{2}\right) \\
\text { and } * \text { p value }\end{array}$} \\
\hline & $\begin{array}{l}\text { MBL production } \\
\text { present, } \mathrm{N}(\%)\end{array}$ & $\begin{array}{l}\text { MBL production } \\
\text { absent, N (\%) }\end{array}$ & $\begin{array}{l}\text { Total isolates, } \\
\mathbf{N}(\%)\end{array}$ & \\
\hline MBL production present, $\mathbf{N}(\%)$ & $37(75.5 \%)$ & $12(24.5 \%)$ & $49(100 \%)$ & \multirow{3}{*}{$\begin{array}{l}\chi^{2}=24.344 \\
p<0.001\end{array}$} \\
\hline MBL production absent, N (\%) & $0(0 \%)$ & $13(100 \%)$ & $13(100 \%)$ & \\
\hline Total isolates, $\mathbf{N}(\%)$ & $37(59.7 \%)$ & $25(40.3 \%)$ & $62(100 \%)$ & \\
\hline
\end{tabular}

\begin{tabular}{|c|c|c|c|c|}
\hline Organisms & $\begin{array}{c}\text { MBL } \\
\text { producers, } \\
\text { N }(\%)\end{array}$ & $\begin{array}{c}\text { Non-MBL } \\
\text { producers, } \\
\text { N }(\%)\end{array}$ & $\begin{array}{c}\text { Total isolates } \\
\text { tested } \\
\text { N }(\%)\end{array}$ & $\begin{array}{c}\text { Chi- Square }\left(\chi^{2}\right) \\
\text { value and } * p \\
\text { value }\end{array}$ \\
\hline Pseudomonas aeruginosa & $40(21.9 \%)$ & $143(78.1 \%)$ & $183(100 \%)$ & \multirow{3}{*}{$\begin{aligned} \chi^{2} & =0.508 \\
p & =0.476\end{aligned}$} \\
\hline Acinetobacter species & $09(17.3 \%)$ & $43(82.7 \%)$ & $52(100 \%)$ & \\
\hline Total isolates & $49(20.9 \%)$ & $186(79.1 \%)$ & $235(100 \%)$ & \\
\hline
\end{tabular}

\begin{tabular}{l|l|l|l|l|}
\hline \multirow{2}{*}{ Table.4 Distribution of organisms according to their MBL producing status and source of samples } \\
tested (N= 235)
\end{tabular}

\begin{tabular}{|c|c|c|c|c|}
\hline Samples tested & $\begin{array}{l}\text { MBL } \\
\text { producers } \\
\text { N }(\%)\end{array}$ & $\begin{array}{l}\text { Non-MBL } \\
\text { producers } \\
\text { N }(\%)\end{array}$ & Total isolates $\mathbf{N}(\%)$ & $\begin{array}{l}\text { Chi- Square }\left(\chi^{2}\right) \\
\text { value } \\
\text { and *p value }\end{array}$ \\
\hline Pus & $31(33.7 \%)$ & $61(66.3 \%)$ & $92(100 \%)$ & \multirow{6}{*}{$\begin{array}{l}\chi^{2}=17.489 \\
p=0.002\end{array}$} \\
\hline Urine & $04(8.5 \%)$ & $43(91.5 \%)$ & $47(100 \%)$ & \\
\hline Blood & $01(4.8 \%)$ & $20(95.2 \%)$ & $21(100 \%)$ & \\
\hline Sputum & $08(18.6 \%)$ & $35(81.4 \%)$ & $43(100 \%)$ & \\
\hline Catheter Tip & $05(15.6 \%)$ & $27(84.4 \%)$ & $32(100 \%)$ & \\
\hline Total & $49(20.9 \%)$ & $186(79.1 \%)$ & $235(100 \%)$ & \\
\hline
\end{tabular}


Table.6 Comparative evaluation of in vitro antibiotic susceptibility pattern of MBL producing and nonMBL producing isolates of Pseudomonas aeruginosa for the commonly used antibiotics $(\mathbf{N}=183)$

\begin{tabular}{|c|c|c|c|}
\hline Antibiotics tested & $\begin{array}{l}\text { Percentage of } \\
\text { susceptible } \\
\text { isolates among } \\
\text { MBL producers } \\
(\mathrm{N}=\mathbf{4 0})\end{array}$ & $\begin{array}{l}\text { Percentage of } \\
\text { susceptible isolates } \\
\text { among Non-MBL } \\
\text { producers } \quad(\mathrm{N}= \\
\text { 143) }\end{array}$ & $\begin{array}{l}\text { Chi-Square }\left(\chi^{2}\right) \text { and } * p \\
\text { value }\end{array}$ \\
\hline Amikacin & $15.0 \%$ & $64.3 \%$ & $\chi^{2}=30.586, p<0.001$ \\
\hline Gentamicin & $10.0 \%$ & $54.5 \%$ & $\chi^{2}=25.079, p<0.001$ \\
\hline Piperacillin & $0 \%$ & $26.6 \%$ & $\chi^{2}=13.415, p<0.001$ \\
\hline Piperacillin-tazobactam & $5.0 \%$ & $74.1 \%$ & $\chi^{2}=61.751, p<0.001$ \\
\hline Ceftazidime & $0 \%$ & $18.9 \%$ & $\chi^{2}=8.860, p=0.003$ \\
\hline Cefepime & $0 \%$ & $51.7 \%$ & $\chi^{2}=34.752, p<0.001$ \\
\hline Aztreonam & $30.0 \%$ & $21.0 \%$ & $\chi^{2}=1.438, p=0.230$ \\
\hline Ciprofloxacin & $7.5 \%$ & $41.3 \%$ & $\chi^{2}=15.902, p<0.001$ \\
\hline Imipenem & $0 \%$ & $96.5 \%$ & $\chi^{2}=156.979, p<0.001$ \\
\hline Meropenem & $0 \%$ & $98.6 \%$ & $\chi^{2}=171.848, p<0.001$ \\
\hline Colistin & $100 \%$ & $100 \%$ & NA \\
\hline Polymyxin B & $100 \%$ & $100 \%$ & NA \\
\hline
\end{tabular}

Fig.1 Shows an isolate with zone of inhibition of imipenem-EDTA disk $\geq 7 \mathrm{~mm}$ more than that of imipenem disk alone, hence, it was considered as MBL producer

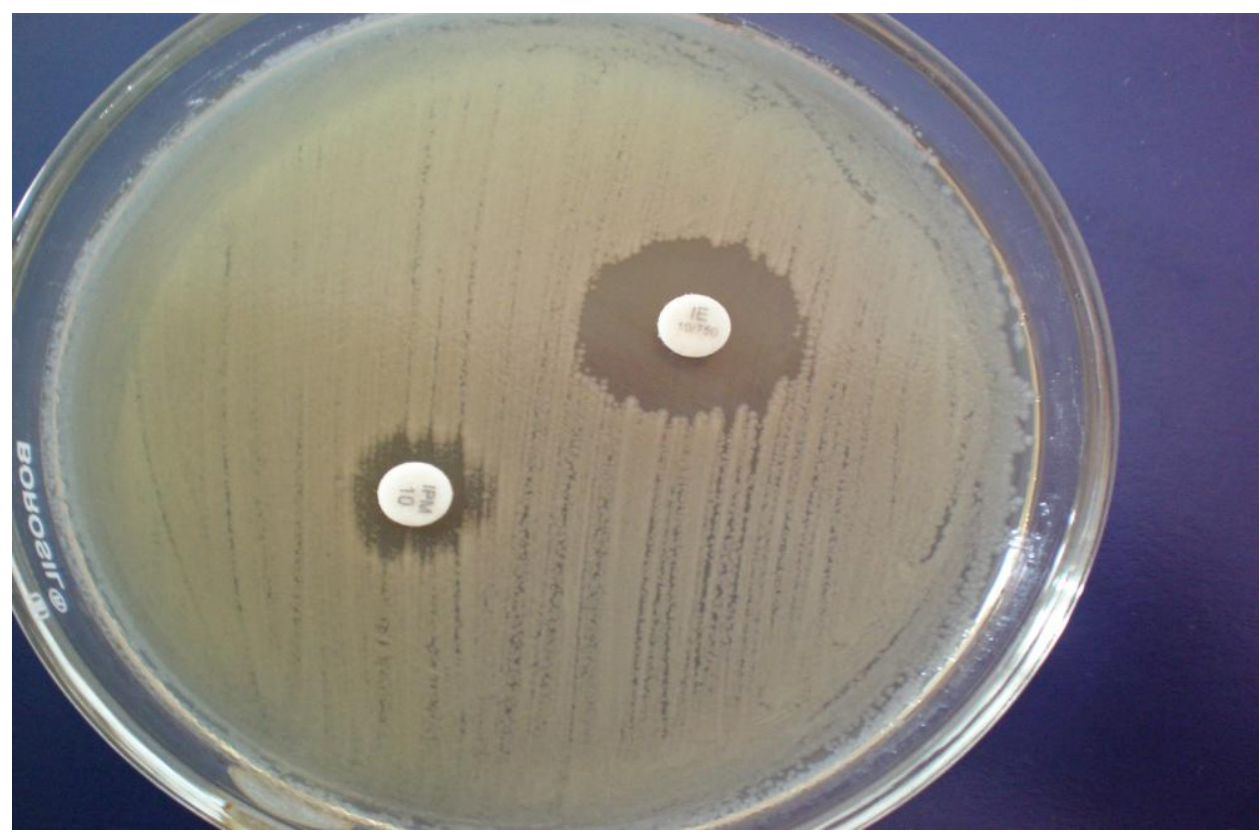




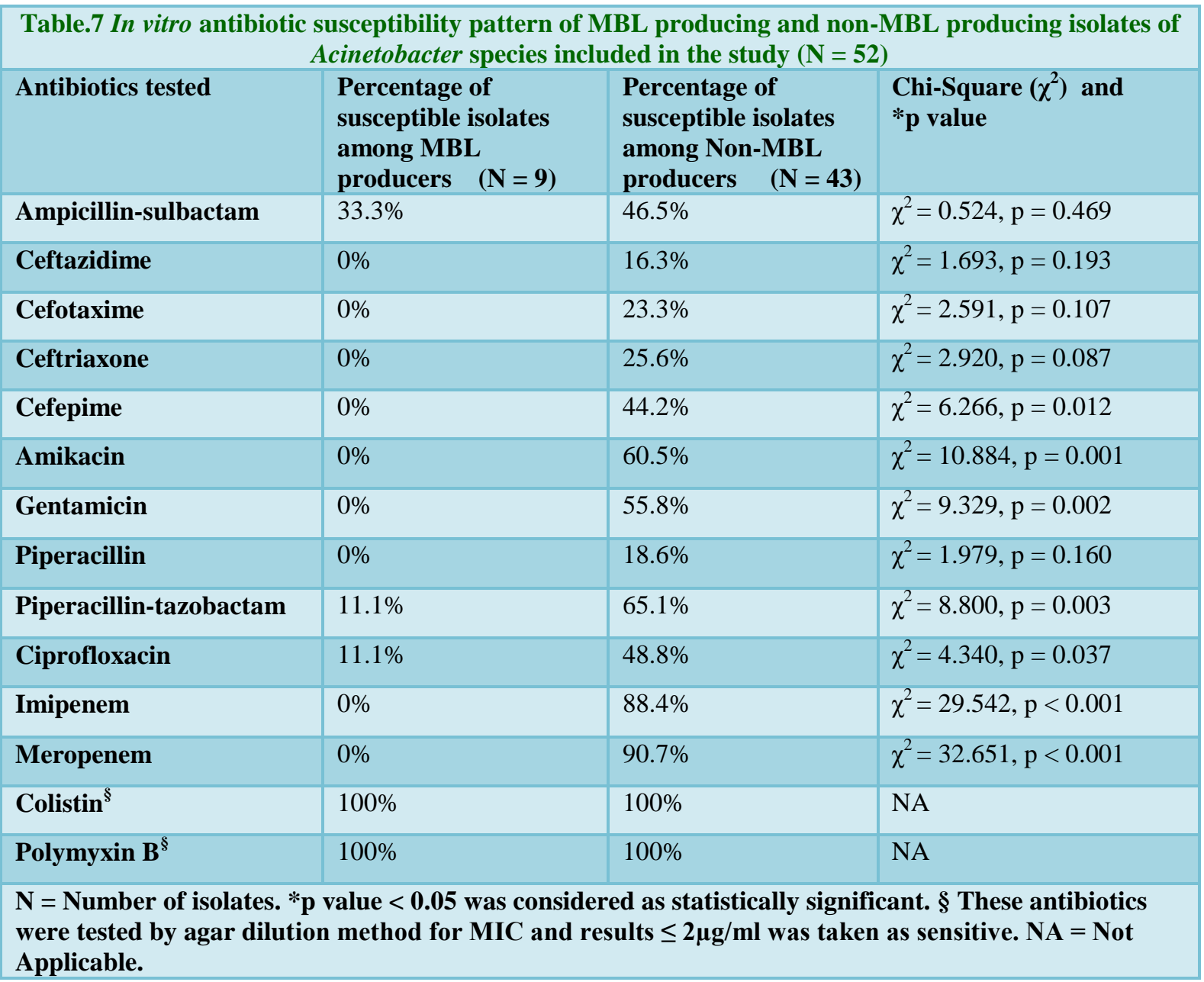

Fig.2 Shows that the test strain produces the enzyme and allows the growth of the carbapenem susceptible

Escherichia coli ATCC 25922 strain towards the imipenem disk, thereby causing the appearance of distorted inhibition zone of imipenem after overnight incubation, hence, the test strain was interpreted as modified Hodge test positive and an MBL producer

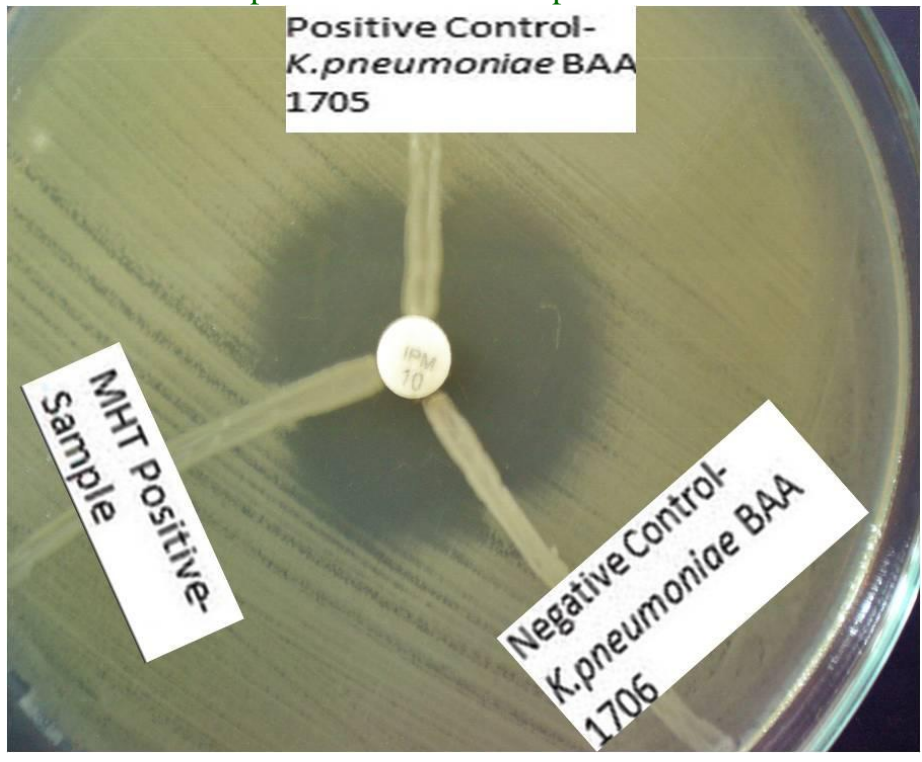


Fig.3 Shows the distribution of isolates of Pseudomonas aeruginosa and Acinetobacter species resistant to carbapenems tested, hence, they were considered as screen positives for MBL production

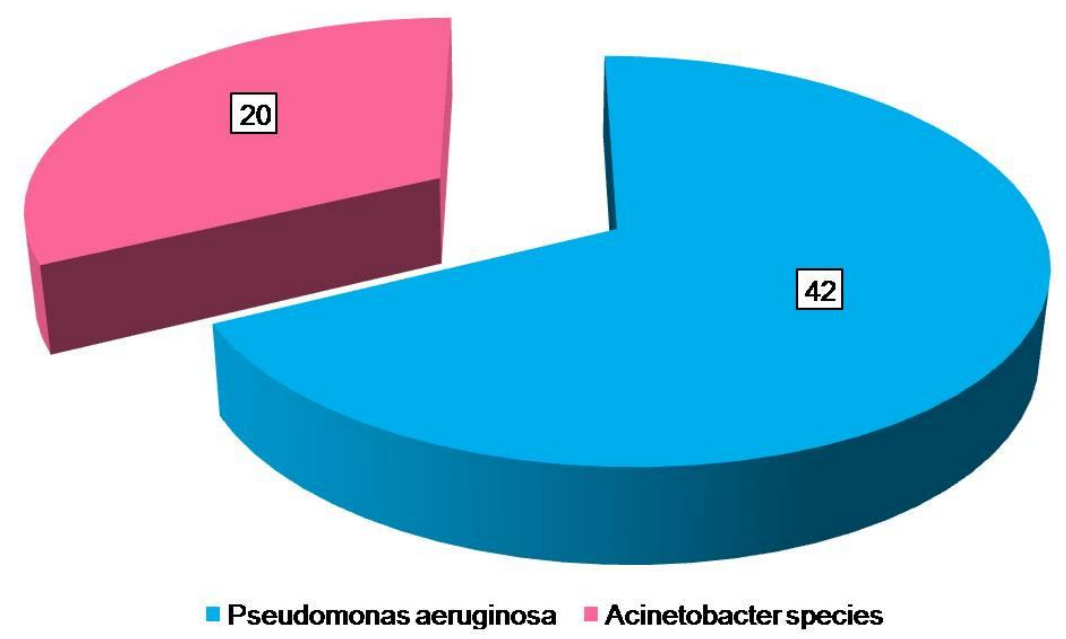

In our study, out of 235 isolates, 62 were found to be carbapenem resistant, these isolates when subjected to confirmatory tests yielded positive results of MBL producing organisms among $79.0 \%$ isolates by CDT and $59.7 \%$ isolates by MHT. Similarly a study done in Meerut detected more number of MBL producers among organisms using CDT as compared to MHT (Chauhan et al., 2015). In our study, the prevalence of MBL production was found to be higher among isolates of Pseudomonas aeruginosa (21.9\%) as compared to those among Acinetobacter species $(17.3 \%)$. Our this finding is in agreement with another study done in Gujarat which also detected higher prevalence of MBL production among isolates of Pseudomonas aeruginosa (11.42\%) as compared to those among Acinetobacter species (10.40\%) (Ahir et al., 2012). Another study done in Kolkata, also detected higher prevalence of MBL production among Pseudomonas aeruginosa isolates (41\%) as compared to Acinetobacter species (22\%) (Rit et al., 2013). However, in contrast to our finding, a study from Mumbai detected higher prevalence of $\mathrm{MBL}$ production among isolates of Acinetobacter species (36\%) as compared to Pseudomonas aeruginosa (28.57\%) (De et al., 2010).

In the present study, the prevalence of MBL producing organisms was found to be higher among isolates from inpatients $(26.1 \%)$ in comparison to those from outpatients $(9.5 \%)$. However, detection of MBL production in an organism isolated from samples received from outpatients is a matter of concern as such strains may spread rapidly into the community and cause therapeutic problem.

In our study, most of the MBL producers were isolated from pus samples (33.7\%), followed by sputum (18.6\%), and least from blood $(4.8 \%)$. Our this finding corroborates well with another study from Maharashtra which also detected maximum MBL producers isolated from pus samples $(36.8 \%)$, followed by tracheal secretions (26.3\%), urine (15.9\%) and least from blood and ascitic fluid (10.6\% each) (Deshmukh et al., 2011).

In the present study, antibiotic susceptibility profile showed that MBL producers were 
multidrug resistant, with both Pseudomonas aeruginosa and Acinetobacter species isolates being highly resistant to all the drugs tested except colistin and polymyxin B to which both were still $100 \%$ sensitive. This finding corroborates with another study from Kolkata which detected that MBL producing isolates were multidrug resistant except for colistin (100\% sensitive) and for polymyxin-B (92\% sensitivity by Pseudomonas aeruginosa and $85 \%$ sensitivity by Acinetobacter species) (Rit et al., 2013). Another study from Maharashtra also detected that all the MBL producers were $100 \%$ sensitive to colistin (Deshmukh et al., 2011). Similarly, various workers have reported polymyxin $\mathrm{B}$ as the most sensitive drug for MBL producers (Pandya et al., 2011). But polymyxin B being a very toxic drug should not be used as a monotherapy. It can be combined with an appropriate aminoglycoside. Aztreonam is the drug of choice for MBL producing Pseudomonas aeruginosa (Walsh et al., 2005). Combination therapy is often employed in treatment of multidrug-resistant Acinetobacter species (De et al., 2010). Imipenem or meropenem combined with ampicillin-sulbactam is found to be active against carbapenem-resistant as well as MBL-positive strains of Acinetobacter species (Perez et al., 2007).

To conclude, our finding shows that there are significant numbers of $\mathrm{MBL}$ producing isolates with multidrug resistance not only among hospitalized patients but also among outpatients. This situation prompts the early detection of MBL-producing isolates which would help in reduction of mortality rates of patients and also to avoid the intra-hospital and inter-hospital dissemination of such strains. Detection of MBL is a challenge for routine microbiology laboratories, since there are no standardized methods for MBL detection. However, as detected in our study, CDT (Imipenem-EDTA) is the most convenient phenotypic method for detection of MBL production in Gram negative bacilli with high sensitivity and its advantage is that it is also less time consuming, technically less demanding as compared to MHT, therefore, less cumbersome to perform in routine microbiological laboratories. Also, one must enforce strict infection control policies and antibiotic policies for judicious use of carbapenems and other broad spectrum antibiotics in order to reduce the escalation of such resistant organisms.

Conflict of Interest: None declared.

\section{References}

Ahir HR, Patel PH, Berry RA, Parmar R, Soni ST, Shah PK, Vegad MM, Patil S. Prevalence of Metallo- $\beta$-lactamases producing Pseudomonas and Acinetobacter species in Tertiary care teaching hospital, Gujarat. Intern $\mathbf{J}$ Microbiol Res 2012;4:322-5.

Altun S, Tufan ZK, Yagci S, Onde U, Bulut C, Kinikl S, Demiroz AP. Extended spectrum beta-lactamases, AmpC and metallo beta-lactamases in emerging multi-drug resistant gram-negative bacteria in intensive care units. Open Access Sci Rep 2013;2:1-4.

Andre B, Philippe B, Nathalie G, Catherine D, Dominique D, Guillaume A, Edouard B. Phenotypic screening of carbapenemases and associated $\beta$ Lactamases in carbapenem-resistant Enterobacteriaceae. J Clin Microbiol 2012; 50: 1295-1302.

Bennett PM. Integrons and gene cassettes; A genetic construction kit for bacteria. Antimicrob Agents Chemother1999; 43:1-4.

Bush K, Jacoby GA, Medeiros A. A functional classification scheme for beta lactamase and its correlation with molecular structure. Antimicrob Agents Chemother1995; 39:1211-33. 
Chauhan K, Pandey A, Asthana AK, Madan M. Evaluation of phenotypic tests for detection of Klebsiella pneumoniae carbapenemase and metallo-betalactamase in clinical isolates of Escherichia coli and Klebsiella species. Indian J Pathol Microbiol 2015; 58:31-5.

Clinical and Laboratory Standards Institute (CLSI). Performance standards for antimicrobial susceptibility testing; $26^{\text {th }}$ edition. CLSI supplement M100S. Clinical and Laboratory Standards Institute, 950 West Valley Road, Suite 2500, Wayne, Pennsylvania 19087 USA, 2016.

Crichton PB. Enterobacteriaceae: Escherichia, Klebsiella, Proteus and other genera. In: Collee JG, Fraser AG, Marmion BP, Simmons A, editors. Mackie and McCartney Practical Medical Microbiology. 14th ed. Edinburgh: Churchill Livingstone; 2006; p.368-70.

De AS, Kumar SH, Baveja SM. Prevalence of metallo- $\beta$-lactamase producing Pseudomonas aeruginosa and Acinetobacter species in intensive care areas in a tertiary care hospital. Indian J Cri Care Med 2010;14:217-19.

Deshmukh DG, Damle AS, Bajaj JK, Bhakre JB and Patwardhan NS. Metallo- $\beta$ lactamase-producing clinical isolates from patients of a Tertiary Care Hospital. J Lab Physicians 2011;3: 93-97.

El-Din RAA, El-Bassat H, El-Bedewy M, ElMohamady H. Prevalence of metallo$\beta$-lactamases producers among carbapenem-resistant Acinetobacter baumannii strains isolated from diabetic foot ulcers. African $\mathbf{J}$ Microbiol Res 2014;8:1580-5.

Galani I, Rekatsina PD, Hatzaki D, Plachouras D, Souli M, Giamarellou H. Evaluation of different laboratory tests for the detection of metalloBlactamase production in Enterobacteriaceae. J Antimicrob Chemother 2008;61 548-53.

Jones RN, Sader HS, Fritsche TR, and Rhomberg PR. Carbapenem susceptibility discords among Acinetobacter isolates. Clin Infect Dis 2006; 42:158.

Lee K, Chong Y, Shin HB, Kim YA, Yong D, Yum JH. Modified Hodge test and EDTA-disk synergy tests to screen metallo- $\beta$-lactamase-producing strains of Pseudomonas and Acinetobacter species. Clin Microbiol Infect 2001; 7: 88-91.

Pandya NP, Prajapati SB, Mehta SJ, Kikani KM, Joshi PJ. Evaluation of various methods for detection of metallo- $\beta$ lactamase (MBL) production in gramnegative bacilli. Int $\mathrm{J}$ Biol Med Res 2011;2:775-7.

Perez F, Hujer AM, Hujer KM, Decker BK, Rather PN, Bonomo RA. Global challenge of multidrugresistant Acinetobacter baumanii. Antimicrob Agents Chemother. 2007;51:3471-84.

Purohit M, Mendiratta D K, Deotale V S, Madhan M, Manoharan A, Narang P. Detection of metallo- $\beta$-lactamases producing Acinetobacter baumannii using microbiological assay, disc synergy test and PCR. Indian J Med Microbiol 2012; 30:456-61.

Rit K, Chakraborty B, Dey R, Chakrabarty P, Naha A, Saha R. Prevalence of Pseudomonas aeruginosa and Acinetobacter spp producing metallo$\beta$-lactamase in a tertiary care hospital. J Dr NTR Univ Health Sci 2013;2:1821.

Saha R, Jain S, Kaur IR. Metallo betalactamase producing pseudomonas species-a major cause of concern among hospital associated urinary tract infection. J Indian Med Assoc 
2010; 108:344-8.

Sarkar B, Biswas D, Prasad R, Sharma JP. A clinicomicrobiological study on the importance of Pseudomonas in nosocomially infected ICU patients, with special reference to metallo beta 1-lactamase production. Indian $\mathbf{J}$ Pathol Microbiol 2006; 49: 44-8.

Senda K, Arakawa Y, Nakashima K, Ito H, Ichiyama S, Shimokata K, Kato N, Ohta M. Multifocal outbreaks of metallo-beta-lactamase producing Pseudomonas aeruginosa resistant to broad-spectrum beta lactams, including carbapenems. Antimicrob Agents Chemother 1996; 40:349-53.
Rajeshwari KG, Kulkarni M. Detection of Metallo-beta lactamase production in clinical isolates of Nonfermentative Gram negative bacilli. IOSR-Journal of Den Med Sciences 2015;14:43-8.

Varaiya, N. Kulkarni, M. Kulkarni, P. Bhalekar, J Dogra. Incidence of metallo beta lactamase producing Pseudomonas aeruginosa in ICU patients. Indian J Med Res 2008; 127:398-402.

Walsh TR, Toleman MA, Poirel L, Nordmann P. Metallo- $\beta$-lactamases: The quiet before the storm? Clin Microbiol Rev 2005;18:306-25.

\section{How to cite this article:}

Noor Jahan, Razia Khatoon and Mohd Rashid. 2018. Phenotypic Evaluation of Prevalence of Metallo-Beta-Lactamase (MBL) Production among Clinical Isolates of Pseudomonas aeruginosa and Acinetobacter Species in a Tertiary Care Hospital of North India. Int.J.Curr.Microbiol.App.Sci. 7(07): 1799-1809. doi: https://doi.org/10.20546/ijcmas.2018.707.213 